\title{
Atorvastatin protects against contrast-induced nephropathy via anti-apoptosis by the upregulation of Hsp27 in vivo and in vitro
}

\author{
XUYU HE, JUNQING YANG, LIWEN LI, HONG TAN, YING WU, PENG RAN, \\ SHUO SUN, JIYAN CHEN and YINGLING ZHOU
}

\begin{abstract}
Department of Cardiology, Guangdong Provincial Cardiovascular Institute, Guangdong General Hospital, Guangdong Academy of Medical Sciences, Guangzhou, Guangdong 510080, P.R. China
\end{abstract}

Received August 31, 2015; Accepted September 12, 2016

DOI: $10.3892 / \mathrm{mmr} .2017 .6251$

\begin{abstract}
Contrast-induced nephropathy (CIN) is an iatrogenic acute renal failure occurring following the intravascular injection of iodinated radiographic contrast medium. However, the regulatory mechanisms for CIN remain to be fully elucidated. The present study aimed to investigate whether atorvastatin protects against CIN via anti-apoptotic effects by the upregulation of Hsp27 in vivo and in vitro. To determine whether atorvastatin attenuated CIN, the inflammatory response and apoptosis in vivo and in vitro, a rat model of iopamidol-induced CIN was used, and human embryonic proximal tubule (HK2) cell damage was assessed. The rats were assigned into four groups ( $\mathrm{n}=10$ per group), as follows: Control rats; rats+atorvastatin; rats + iopamidol; rats+iopamidol+atorvastatin. In vitro, the HK2 cells were treated with iopamidol in the presence or absence of atorvastatin, heat shock protein (Hsp)27 small interfering (si) RNA or pcDNA3.1-Hsp27. The renal tissues were examined histopathologically and collected for western blot analysis. The results showed that atorvastatin ameliorated the apoptosis and deterioration of renal function $(\mathrm{P}<0.05)$. Furthermore, atorvastatin reduced the iopamidol-induced activity of $\mathrm{B}$ cell lymphoma-2 (Bcl-2)-associated X protein (Bax)/caspase-3 and increased the expression of Bcl-2 in vivo and in vitro. Notably, following treatment with Hsp27 siRNA or pcDNA3.1-Hsp27, it was found that iopamidol enhanced or weakened the upregulation of Bax/caspase-3 and downregulation of Bcl-2 in the HK2 cells, respectively. The results of the present study suggested that atorvastatin protected against contrast-induced
\end{abstract}

Correspondence to: Dr Jiyan Chen or Dr Yingling Zhou, Department of Cardiology, Guangdong Provincial Cardiovascular Institute, Guangdong General Hospital, Guangdong Academy of Medical Sciences, 106 Zhongshan Road, Guangzhou, Guangdong 510080, P.R. China

E-mail: gdphospital@163.com

E-mail: zhouyingling852@163.com

Key words: contrast-induced nephropathy, heat shock protein 27, nephropathy, anti-apoptosis renal tubular cell apoptosis through the upregulation of Hsp27 in vivo and in vitro.

\section{Introduction}

Contrast-induced nephropathy (CIN) is an acute renal failure, which occurs following the use of contrast medium (CM) for diagnostic imaging and interventional procedures, including angiography. It is the third leading cause of hospital-acquired renal failure worldwide (1). There is no specific therapy or standard preventive procedure, with the exception of hydration and minimizing the dosage of $\mathrm{CM}$, which do not provide optimal prevention $(2,3)$. In previous years, several clinical investigations have been performed, and it has been confirmed that CIN results in increased medical costs, prolonged admissions, disease deterioration, dialysis, long-term decline in renal function and associated mortality rates (4-6). Thus, it is important and urgent to improve current understanding of the mechanism by which CIN occurs and develop novel preventive therapies.

Apoptosis is considered to be a characteristic of acute kidney injury, including ischemia and anoxia, inflammatory reactions and damage from drug toxicity (3). The apoptosis of renal tubular cells is an important pathological alteration in the pathogenesis of CIN, and the occurrence of apoptosis is expected to be important in therapy for improving nephropathy $(7,8)$. Statins exert several effects through their non-lipid-associated mechanisms. These so-called pleiotropic effects encompass several mechanisms, which modify inflammatory responses, endothelial function, plaque stability and apoptosis $(9,10)$. The effectiveness of statin pretreatment in reducing the incidence of contrast-induced acute kidney injury (CIAKI) has been examined in a number of observational and randomized investigations (11-12). There is a general consensus that statins merit further investigation for the prevention of CIAKI. The present study investigated whether atorvastatin can ameliorate iopamidol-induced apoptosis in CIN rats and HK2 cells.

Heat shock proteins (HSPs) are recognized molecular chaperones and function as cytoprotective proteins. HSPs protect other intracellular proteins from denaturation and aggregation, which occur in response to oxidative stress (13). Hsp27 is a ubiquitous HSP, the expression of which is induced 
in response to a wide variety of unfavorable physiological and environmental conditions $(13,14)$. Classically, the activation of the expression of Hsp27 occurs be due to an increase in the total protein expression of Hsp27 and/or due to increased activation of an upstream kinase, which phosphorylates Hsp27 (13). It has been reported that $\mathrm{Hsp} 27$ protect cells from otherwise pathological circumstances, predominantly through their involvement in pathways of cell death, including necrosis and apoptosis $(13,14)$. However, the intracellular signaling modulation of Hsp27 in CIN remains to be elucidated. Therefore, the present study was designed to determine whether atorvastatin can protect against CIN due to anti-apoptotic effects and to identify the possible mechanisms of Hsp27 regulation.

\section{Materials and methods}

Animals. All experimental procedures were performed according to the Guide for the Care and Use of Laboratory Animals (15) and were in compliance with the guidelines specified by the Chinese Heart Association policy on research in animal use and the Public Health Service policy on the use of laboratory animals.

Sprague-Dawley rats were used in the present study, which, in addition to the basal diet used, were purchased from Sun Yat-Sen University Laboratory Animal Centre (Guangzhou, China). A total of 40 male rats weighing between 280 and $320 \mathrm{~g}$ were used in the experiment. The rats were housed in multiple mouse racks and acclimated for 1 week in groups (five mice per cage) with free access to a mycotoxin-free diet and tap water. The rats were housed at a constant temperature $\left(25 \pm 2^{\circ} \mathrm{C}\right)$ and constant humidity $(60 \pm 5 \%)$, with artificial lighting every $12 \mathrm{~h}$.

The rats were acclimated for 7 days and then divided into four groups ( $n=10 /$ group), as follows: Control rats (CR); rats+atorvastatin $(20 \mathrm{mg} / \mathrm{kg} / \mathrm{day} ; \mathrm{CA})$; rats + iopamidol $(\mathrm{CM})$; rats+iopamidol+atorvastatin (20 mg/kg/day; CMA). CIN was induced using nonionic low-osmolar iopamidol, as previously described (16). Following a 16-h dehydration period, the iopamidol (Shanghai Bracco Sine Pharmaceutical Corp. Ltd., Shanghai, China) was administered intravenously into the internal carotid artery for $5 \mathrm{~min}$ at a dose of $2.9 \mathrm{~g}$ iodine $/ \mathrm{kg}$ body weight. The control rats received an equal volume of normal saline. The plasma and kidney samples were collected, and the rats were sacrificed by intraperitoneal injection with $10 \%$ chloral hydrate $(300 \mathrm{mg} / \mathrm{kg})$ and subsequently sacrificed. 7 days post-iopamidol injection. Atorvastatin was donated by Pfizer, Inc. (New York, NY, USA). Iopamidol was obtained from Bracco S.p.A. (Milan, Italy).

The experimental animals received no water for $16 \mathrm{~h}$ prior to injection of the left internal carotid artery with contrast agents, $0.3 \%$ pentobarbital sodium and $1 \mathrm{ml} / 100 \mathrm{~g}$ anesthesia. The iopamidol 370 (2.9 g iodine/ $\mathrm{kg} ; 5$-min injection) was injected through the left internal carotid artery, whereas the animals in the control group received saline. The CMA group was treated with atorvastatin $(20 \mathrm{mg} / \mathrm{kg} / \mathrm{day}$, dissolved in $2 \mathrm{ml}$ of saline) 1 day prior to $\mathrm{CM}$ injection and 3 days following $\mathrm{CM}$ injection. The CA group was treated with atorvastatin ( $20 \mathrm{mg} / \mathrm{kg} /$ day, dissolved in $2 \mathrm{ml}$ of saline) for 4 days without CM injection. Blood was collected from the left femoral artery, and the serum was separated by $12,000 \mathrm{x} \mathrm{g}$ centrifugation for $10 \mathrm{~min}$ at $4^{\circ} \mathrm{C}$ to assess renal function. The kidneys were removed for biochemical and histopathological investigations.

Determination of creatinine in the serum and urine. The concentrations of creatinine (Scr) and urea (BUN) in the serum and urine were analyzed as indicators of injured glomerular function. Creatinine clearance $(\mathrm{ml} / \mathrm{min})$ was calculated according to the following formula: UV / P, where $\mathrm{U}$ represents creatinine concentration in the urine $(\mathrm{mg} / \mathrm{dl})$, $\mathrm{V}$ represents urine volume $(\mathrm{ml} / \mathrm{min})$ and $\mathrm{P}$ represents serum creatinine $(\mathrm{mg} / \mathrm{dl})$. The fractional excretion of sodium was calculated according to the following formula: (Urine sodium / serum sodium) x (serum creatinine / urine creatinine) $\mathrm{x}$ 100. Urinary kidney injury molecule-1 (uKIM-1) was measured using ELISA kits (R\&D Systems, Inc., Minneapolis, MN, USA).

Assessment of renal apoptosis in CIN rats. TUNEL staining for cell apoptosis in renal tissues was used to evaluate the extent of apoptosis according to the manufacturer's protocol (Roche Diagnostics, Mannheim, Germany). Tissues were imaged using a fluorescent microscope.

Culture conditions and reagents. The HK2 cells lines were grown in a Dulbecco's modified Eagle's medium (DMEM)-F12 mixture (Santa Cruz Biotechnology, Inc., Santa Cruz, CA, USA) with $10 \%$ heat-inactivated fetal bovine serum (Santa Cruz Biotechnology, Inc.), 2 mmol/l L-glutamine and $100 \mathrm{U} / \mathrm{ml}$ penicillin-streptomycin at $37^{\circ} \mathrm{C}$ with $5 \% \mathrm{CO}_{2}$. The cells were routinely passaged when $80-85 \%$ confluent. The media, sera and antibiotics for cell culture were obtained from Sigma-Aldrich; Merck Millipore (Darmstadt, Germany). Prior to treatment, the cells were cultured in DMEM/F12 low glucose $(5 \mathrm{mM})$ for $24 \mathrm{~h}$. Basal $(5 \mathrm{mM})$ glucose culture media was produced as described previously (11).

Protein electrophoresis reagents were obtained from Bio-Rad Laboratories, Inc. (Richmond, VA, USA). The HK2 cells were pretreated with atorvastatin at a dose of $0.2 \mu \mathrm{mol} / 1$ at $37^{\circ} \mathrm{C}$ with $5 \% \mathrm{CO}_{2}$. The dose of atorvastatin was selected according to the standard doses used in cell lines.

Experimental design. When the cells culture was at $\sim 80 \%$ confluence, the cells were serum-starved for $12 \mathrm{~h}$ at $37^{\circ} \mathrm{C}$ with $5 \% \mathrm{CO}_{2}$, following which they were used in the following experiments:

i) Time-course experiments. The HK2 cells ( $~ 80 \%$ confluence) were treated with iopamidol $(200 \mathrm{mg} / \mathrm{ml})$ for $0,4,8,16,24$ or $48 \mathrm{~h}$ at $37^{\circ} \mathrm{C}$ with $5 \% \mathrm{CO}_{2}$. The cells were analyzed using a cell titer proliferation assay and harvested for reverse transcription-quantitative polymerase chain reaction (RT-qPCR) and western blot analyses to determine the expression levels of Hsp27, Bax, Bcl-2 and cleaved/total caspase-3. The cell activity levels were assessed by cell death quantification.

ii) Investigation of the effect of atorvastatin on iopamidol-induced cell apoptosis. The HK2 cells $(\sim 80 \%$ confluence) were treated in the following groups at $37^{\circ} \mathrm{C}$ with $5 \% \mathrm{CO}_{2}$ : Control group; atorvastatin $(0.2 \mu \mathrm{mol} / \mathrm{l})$; iopamidol $(200 \mathrm{mgI} / \mathrm{ml})$; iopamidol $(200 \mathrm{mgI} / \mathrm{ml})+$ atorvastatin $(0.2 \mu \mathrm{mol} / \mathrm{l})$. The cells were harvested for western blot analysis 
to determine the expression levels of Bax, Bcl-2, cleaved/total caspase-3 and Hsp27. The levels of apoptosis were assessed using TUNEL staining.

iii) Investigation of the role of $\mathrm{Hsp} 27$ in mediating iopamidol-induced HK2 cell apoptosis. The HK2 cells ( $\sim 80 \%$ confluence) were treated in the following groups at $37^{\circ} \mathrm{C}$ with $5 \% \mathrm{CO}_{2}$ : Iopamidol $(200 \mathrm{mgI} / \mathrm{ml})$; iopamidol $(200 \mathrm{mgI} / \mathrm{ml})+\mathrm{Hsp} 27$ siRNA (400 pmol); iopamidol $(200 \mathrm{mgI} / \mathrm{ml})+$ pcDNA3.1-Hsp27 $(2 \mu \mathrm{g})$ for $24 \mathrm{~h}$. The expression levels of Bax, Bcl-2 and cleaved/total caspase- 3 was analyzed by western blot analysis. The levels of apoptosis were assessed using TUNEL staining.

Caspase assay. The assay was performed with the use of the Colorimetric CaspACE assay system (Promega Corporation, Madison, WI, USA) according to the manufacturer's protocol. Briefly, the cells were harvested in caspase assay buffer, and proteins were quantified using a Bradford assay. A $50 \mu \mathrm{g}$ quantity of protein was used.

Detection of apoptotic cells using TUNEL staining. A TUNEL kit, purchased from Boehringer Mannheim GmbH (Mannheim, Germany) was used to label the apoptotic cells according to the manufacturer's protocol. The cells with brown particles visible in their nuclei were apoptotic cells. Under a high-power microscope (magnification, x400), five visual fields were randomly collected and analyzed using pathological image software (Motic Med 6.0; Beijing University of Aeronautics and Astronautics, Beijing, China), and the positive cells were counted, with the average value of the five visual fields used as the index of cell apoptosis.

Transient transfection with small interfering (si)RNA. The cells were transfected with Hsp27 siRNA or nontargeting control siRNA using DharmaFECT siRNA transfection reagent according to the manufacturer's protocol (Thermo Fisher Scientific, Inc., Waltham, MA, USA) when at $\sim 80 \%$ confluence for $24 \mathrm{~h}$. The Hsp27 siRNA oligos consisted of three target-specific siRNAs, and were designed and applied as a pool to knock down the gene expression of Hsp27. The target sequences were as follows: Sense 5'-GAG UGG UCG CAG UGG UUA Gtt-3', antisense 5'-CUA ACC ACU GCG ACC ACU Ctt-3'; sense 5'-GAC GAG CUG ACG GUC AAG Att-3', antisense 5'-UCU UGA CCG UCA GCU CGU Ctt-3'; sense 5'-CCA CGC AGU CCA ACG AGA Utt-3', antisense 5'-AUC UCG UUG GAC UGC GUG Gtt-3'. The medium was then replaced with fresh culture medium containing $10 \mathrm{mmol} / \mathrm{l}$ $\mathrm{CA}$ for 0.5 or $24 \mathrm{~h}$, and protein expression was examined using western blot analysis. For the luciferase assay, the cells were transfected with p2xARE/Luc vectors (Promega Corporation) prior to CA treatment.

Construction of the pcDNA3.1-Hsp27 vector. PCR was used to amplify the Hsp27 fragment of the pMD-Hsp27 vector. The 5' primer (5'-AAA GAA TTC ATG ACC GAG CGC CGC GTC-3') and the 3' primer (5'-TTT GGA TCC TTA CTT GGC GGC AGT CTC A-3') were engineered using EcoRI and BamHI restriction sites, respectively. A 50- $\mu 1$ amplification reaction sample containing each primer $(1 \mathrm{mM}), 10 \mu \mathrm{g}$ pMD-Hsp27 vector and 1 unit Pfu DNA polymerase (Tiangen
Table I. Primers for reverse transcription-quantitative polymerase chain reaction analysis.

\begin{tabular}{lll}
\hline Gene & Primer & \multicolumn{1}{c}{ Primer sequence (5'-3') } \\
\hline Bax & Forward & CCC GAG AGGTCT TTT TCC GAG \\
& Reverse & CCA GCC CAT GAT GGTTCT GAT \\
Bcl-2 & Forward & CTT TGA GTTCGG TGG GGT CA \\
& Reverse & GGG CCG TAC AGT TCCACA AA \\
\multirow{2}{*}{ Hsp27 } & Forward & TGC CCT TCT CGC TAC TG \\
& Reverse & ACG CCT TCC TTG GTC TTA \\
\multirow{3}{*}{-actin } & Forward & CAT CTC TTG CTC GAA GTC CA \\
& Reverse & ATC ATG TTT GAG ACC TTC AACA
\end{tabular}

Bcl-2, B cell lymphoma-2, Bax, Bcl-2-associated X protein; Hsp27, heat shock protein 27 .

Biotech (Beijing) Co., Ltd., Beijing, China) was subjected to 30 amplification cycles at $95^{\circ} \mathrm{C}$ for $1 \mathrm{~min}, 60^{\circ} \mathrm{C}$ for $30 \mathrm{sec}$ and $72^{\circ} \mathrm{C}$ for $1 \mathrm{~min} 30 \mathrm{sec}$. Adenylate was added to the 618-bp amplification product at the 3'-end by Taq DNA polymerase, and the fragment was cloned into the pMD18-T vector (Promega Corporation), termed pMD18-T-Hsp27. The pMD18-T-Hsp27 was digested with EcoRI to obtain the Hsp27 fragment, and then cloned to the pcDNA3.1-puro vector, which was digested with $X b a I$ and NheI through flat-end cloning. Restriction enzymes were obtained from Invitrogen; Thermo Fisher Scientific, Inc. The resulting plasmid, pcDNA3.1-Hsp27, was subjected to sequence analysis to confirm the integrity of the inserted element. The pcDNA3.1-puro-XbaI-NheI fragment was self-ligated as a comparison plasmid, termed pcDNA3.1. Lipofectamine $^{\mathrm{TM}} 2000$ (Invitrogen; Thermo Fisher Scientific, Inc.) was used for stable transfection according to the manufacturer's protocol.

RNA isolation and RT-qPCR analysis. Total RNA from the cultured cells was extracted using TRIzol reagent (TransGen Biotech, Beijing, China) according to the manufacturer's protocol. cDNA was synthesized using a SYBR ExScript RT-PCR kit (Toboyo Co., Ltd., Osaka, Japan) according to the protocol provided by the manufacturer. RNA $(1 \mu \mathrm{g})$ was converted into cDNA using a PrimeScript ${ }^{\mathrm{TM}}$ RT reagent kit with gDNA Eraser (Takara Biotechnology Co., Ltd., Dalian, China) according to the manufacturer's protocol. RT-qPCR analysis was performed using SYBR ${ }^{\circledR}$ Premix Ex Taq ${ }^{\mathrm{TM}}$ II (Takara Biotechnology Co., Ltd.) in the ABI PRISM ${ }^{\circledR} 7500$ real-time PCR system (Applied Biosystems; Thermo Fisher Scientific, Inc.). PCR was performed with the cDNA templates in a reaction buffer containing $6.25 \mathrm{pM}$ of each primer with the following standard settings: Denaturation at $95^{\circ} \mathrm{C}$ for $30 \mathrm{sec}$, annealing for $30 \mathrm{sec}$ at $65^{\circ} \mathrm{C}$ or $55^{\circ} \mathrm{C}$ and extension for $30 \mathrm{sec}$ at $72^{\circ} \mathrm{C}$. A total of 30 cycles were performed for amplification. $\beta$-actin was used as an endogenous control. Melting curves were used to evaluate non-specific amplification. The relative expression level was calculated using the $\Delta \Delta \mathrm{Cq}$ method (17). The primer sequences used are presented in Table I. 
Table II. Renal function parameters in the treatment groups.

\begin{tabular}{lcccc}
\hline Parameter & CR & CA & CM & CMA \\
\hline Cr $(\mu \mathrm{mol} / \mathrm{l})$ & $46.00 \pm 4.00$ & $45.00 \pm 6.00$ & $78.00 \pm 6.00^{\mathrm{b}}$ & $48.00 \pm 5.00^{\mathrm{d}}$ \\
BUN $(\mathrm{mmol} / \mathrm{l})$ & $5.00 \pm 1.20$ & $5.20 \pm 1.10$ & $5.60 \pm 3.20$ & $5.40 \pm 1.30$ \\
uKIM-1(ng/ml) & $2.34 \pm 0.07$ & $2.33 \pm 0.10$ & $16.81 \pm 0.14^{\mathrm{a}}$ & $8.42 \pm 0.11^{\mathrm{a}, \mathrm{c}}$ \\
Creatinine clearance $(\mathrm{ml} / \mathrm{min})$ & $2.58 \pm 0.35$ & $2.60 \pm 0.27$ & $1.58 \pm 0.27^{\mathrm{a}}$ & $2.62 \pm 0.26^{\mathrm{c}}$ \\
Urine microprotein $(\mathrm{mg} / \mathrm{dl})$ & $46.50 \pm 10.40$ & $45.70 \pm 11.20$ & $97.50 \pm 13.60^{\mathrm{b}}$ & $51.20 \pm 11.80^{\mathrm{a}, \mathrm{c}}$ \\
\hline
\end{tabular}

Data are presented as the mean \pm standard deviation. ${ }^{\mathrm{a}} \mathrm{P}<0.05$ and ${ }^{\mathrm{b}} \mathrm{P}<0.01$, compared with $\mathrm{CR}$; ${ }^{\mathrm{c}} \mathrm{P}<0.05$ and ${ }^{\mathrm{d}} \mathrm{P}<0.01$, compared with $\mathrm{CM}$. Cr, serum creatinine; BUN, blood urea nitrogen; uKIM-1, urinary kidney injury molecule-1; CR, control rats; CA, atorvastatin (20 mg/kg/day); CM, iopamidol; CMA, iopamidol+atorvastatin (20 mg/kg/day).

Western blot analysis. The western blot analysis was performed as described previously (18). Briefly, $40 \mu \mathrm{g}$ proteins of the total cellular extracts were heated at $99^{\circ} \mathrm{C}$ for $5 \mathrm{~min}$ and loaded onto each lane. Following electrophoresis using $10-12 \%$ SDS-PAGE, the proteins were transferred onto a polyvinylidene difluoride membrane. The membrane was blocked at room temperature for $1 \mathrm{~h}$ in Tris-buffered saline with $0.2 \%$ Tween 20 (TBS-T) containing 5\% skim milk and probed with primary antibodies overnight at $4^{\circ} \mathrm{C}$. The membrane was washed with TBS-T and incubated with a mouse anti-goat monoclonal horseradish peroxidase-conjugated secondary antibody (1:5,000 dilution; cat. no. ab6721; Abcam, Cambridge, $\mathrm{UK})$ for $1 \mathrm{~h}$ at room temperature. Signals were visualized using an enhanced chemiluminescence system (Cell Signaling Technology, Inc. Danvers, MA, USA). The following primary antibodies obtained from Abcam were used: Bax (1:500 dilution; cat. no. ab32503); Bcl-2 (1:500 dilution; cat. no. ab59348); cleaved caspase-3 (1:1,000 dilution; cat. no. ab13847); total caspase-3 (1:1,000 dilution; cat. no. ab52293); Hsp27 (1:500 dilution; cat. no. ab5579); and $\beta$-actin (1:5,000 dilution; cat. no. ab8227).

Statistical analysis. The results of the various proteins were normalized by the corresponding results of $\beta$-actin and are presented as the mean \pm standard deviation from at least five independent experiments, which were performed with cells from different isolations. Statistical analysis was performed using one-way analysis of variance followed by the Student-Newman-Keuls test (SPSS v23.0; IBM SPSS, Armonk, NY, USA). $\mathrm{P}<0.05$ was considered to indicate a statistically significant difference.

\section{Results}

In the present study, 10 rats were randomly divided into each group. All the rats survived until the end of the study.

Effects of atorvastatin on CM-induced renal damage. Atorvastatin decreased levels of $\mathrm{SCr}, \mathrm{BUN}$, urine microprotein and uKIM-1 in the CIN rats. The animals subjected to CIN in the iopamidol groups presented with significantly increased levels of SCr, BUN and UKIM-1, compared with those in the control group. However, the levels of SCr, BUN, urine microprotein and UKIM-1 were all decreased in the rats treated with
Table III. Inflammatory parameters in the treatment groups.

\begin{tabular}{lccc}
\hline Group & TNF- $\alpha(\mathrm{ng} / \mathrm{mg})$ & IL-1 $\beta(\mathrm{g} / \mathrm{mg})$ & IL-6 $(\mathrm{pg} / \mathrm{mg})$ \\
\hline CR & $1.30 \pm 0.12$ & $30.12 \pm 8.23$ & $130.76 \pm 48.77$ \\
CA & $1.26 \pm 0.35$ & $29.76 \pm 6.42$ & $126.13 \pm 48.32$ \\
CM & $2.32 \pm 0.18^{\mathrm{b}}$ & $18.43 \pm 9.24^{\mathrm{a}}$ & $368.0 \pm 47.33^{\mathrm{a}}$ \\
CMA & $1.81 \pm 0.17^{\mathrm{a}, \mathrm{c}}$ & $25.06 \pm 7.98^{\mathrm{a}, \mathrm{c}}$ & $162.45 \pm 50.12^{\mathrm{a}, \mathrm{c}}$ \\
\hline
\end{tabular}

Data are presented as the mean \pm standard deviation. ${ }^{\mathrm{a}} \mathrm{P}<0.05$ and ${ }^{\mathrm{b}} \mathrm{P}<0.01$, compared with $\mathrm{CR} ;{ }^{\mathrm{C}} \mathrm{P}<0.05$, compared with $\mathrm{CM}$. TNF- $\alpha$, tumor necrosis factor- $\alpha$; IL, interleukin; CR, control rats; CA, atorvastatin $(20 \mathrm{mg} / \mathrm{kg} /$ day $)$; CM, iopamidol; CMA, iopamidol+atorvastatin (20 mg/kg/day).

atorvastatin, as shown in Table II. Thus, atorvastatin attenuated the levels of renal function parameters.

As shown in Table III, no significant differences were found between the CR and CA group in the levels of serum tumor necrosis factor (TNF)- $\alpha$, interleukin (IL)-1 $\beta$ and IL-6. Compared with the $\mathrm{CR}$ group, the $\mathrm{CM}$ group had higher serum levels of TNF- $\alpha(\mathrm{P}<0.01)$, IL-1 $\beta(\mathrm{P}<0.05)$ and IL-6 $(\mathrm{P}<0.05)$. However, compared with the $\mathrm{CM}$ group, treatment with atorvastatin reduced the CM-induced increase in TNF- $\alpha(\mathrm{P}<0.05)$, IL-1 $\beta(\mathrm{P}<0.05)$ and IL-6 $(\mathrm{P}<0.05)$. In the CA group, the serum levels of TNF- $\alpha$ and IL-6 were lower, compared with those in the CR group, however, this difference was not statistically significant. TNF- $\alpha$, IL-1 $\beta$ and IL-6 are known inflammatory parameters, which can lead to apoptosis. The findings of the present study suggested that atorvastatin attenuated CM-induced renal damage by modulating inflammatory responses.

Effects of atorvastatin on CM-induced renal cell apoptosis in CIN rats and HK2 cells. As shown in Fig. $1 \mathrm{Aa}$ and b, renal injury was more severe in the CM group, compared with the $\mathrm{CR}$ and $\mathrm{CA}$ groups $(\mathrm{P}<0.01)$, however, the degree of injury was less severe in the CMA group, compared with the CM group $(\mathrm{P}<0.05)$, suggesting that atorvastatin significantly protected the kidney against CIM.

The TUNEL staining (Fig 1B) was also more intense in the $\mathrm{CM}$ group, compared with either the $\mathrm{CR}$ or CA groups. The percentage of apoptotic cells was lower in the CR group, 
$\mathbf{A}_{\mathbf{a}}$

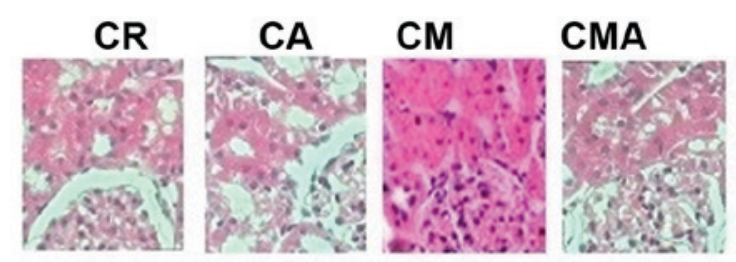

B

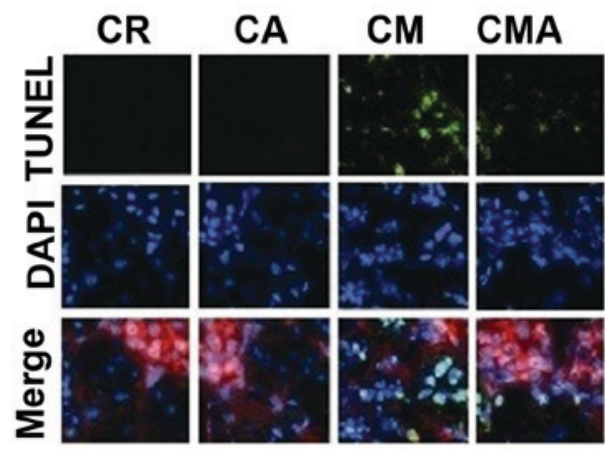

b

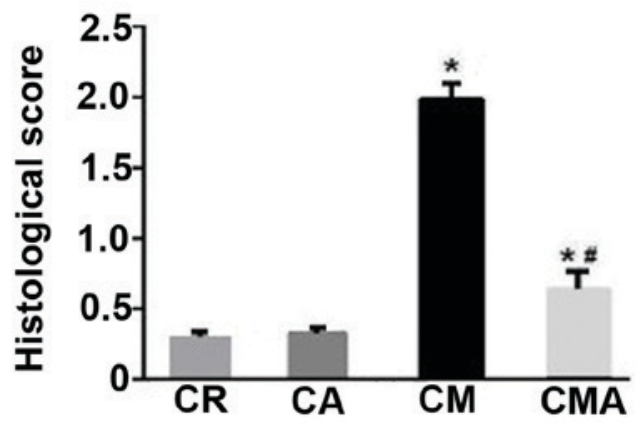

C

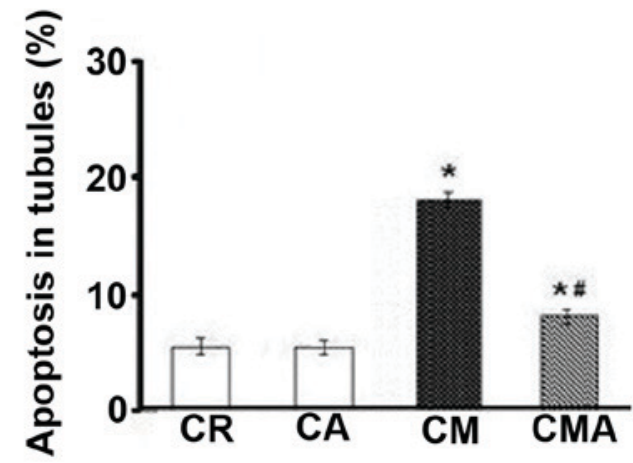

Figure 1. Effects of atorvastatin on iopamidol-induced renal cell damage. (Aa) Light microscopy was used to examine renal tissue from rats stained with HE (magnification, x200). (Ab) Representative images of HE staining are shown with a (Ab) graph of the results from 10 separate experiments. (B) Representative images of TUNEL-positive renal tubular cells (magnification, x400). (C) Graph of the numbers of TUNEL-positive renal tubular cells in the tubule. Data are presented as the mean \pm standard error of the mean ( $\mathrm{P}<0.05$, compared with $\mathrm{CR}$; ${ }^{"} \mathrm{P}<0.05$, compared with $\left.\mathrm{CM}\right)$. HE, hematoxylin and eosin; $\mathrm{CR}$, control rats; CA, atorvastatin (20 mg/kg/day); CM, iopamidol; CMA, iopamidol+atorvastatin (20 mg/kg/day).

A

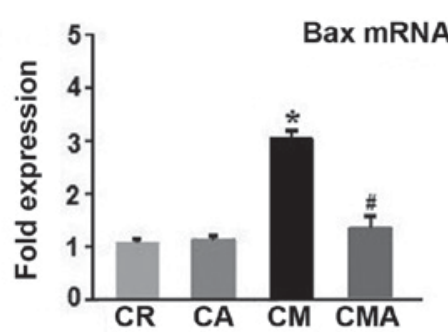

C

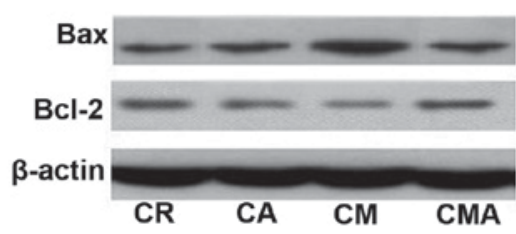

$\mathbf{D}_{\mathbf{a}}$

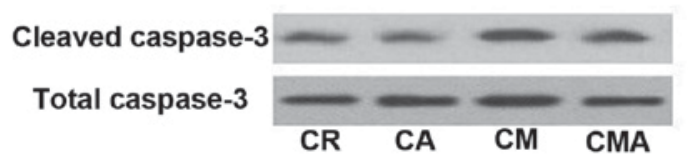

B
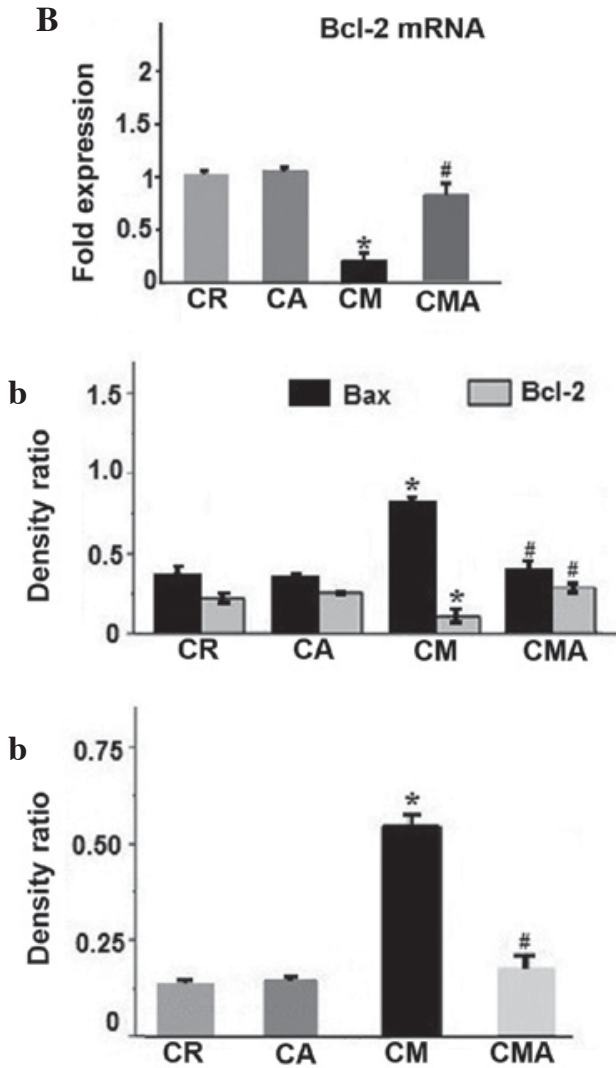

Figure 2. Effects of atorvastatin on CM-induced protein expression levels of Bax/Bcl-2 and cleaved/total caspase-3 in CIN rats. (A) RT-qPCR analysis results for the mRNA expression of Bax. (B) RT-qPCR analysis results for the mRNA expression of Bcl-2. (Ca) Western blot for Bax/Bcl-2 proteins with a (Cb) graph of the results from 10 separate experiments. (Da) Western blot results for cleaved/total caspase-3 proteins are shown, with a (Db) graph of the results from 10 separate experiments. Data are presented as the mean \pm standard error of the mean $\left({ }^{*} \mathrm{P}<0.05\right.$, compared with $\mathrm{CR} ;{ }^{*} \mathrm{P}<0.05$, compared with CM). RT-qPCR, reverse transcription-quantitative polymerase chain reaction; Bcl-2, B cell lymphoma-2, BAX, Bcl-2-associated X protein;CR, control rats; CA, atorvastatin (20 mg/kg/day); CM, iopamidol; CMA, iopamidol+atorvastatin (20 mg/kg/day). 
A

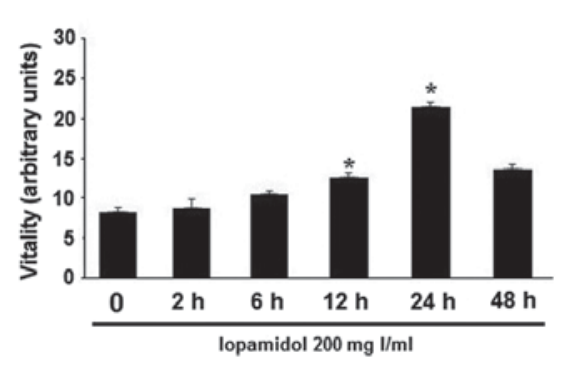

D a

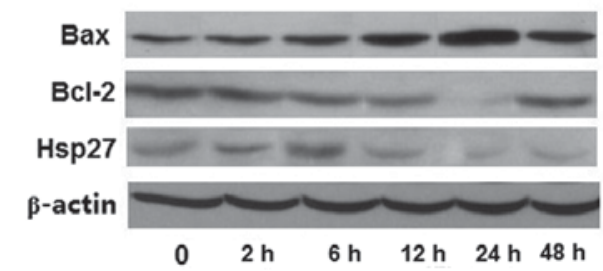

$\mathbf{E}$

a

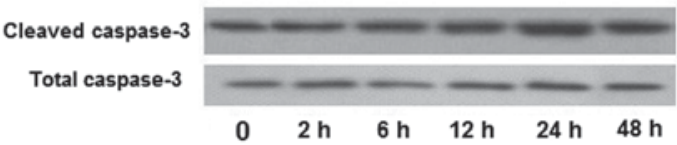

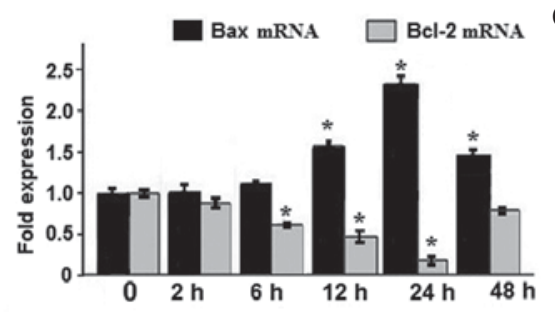

C

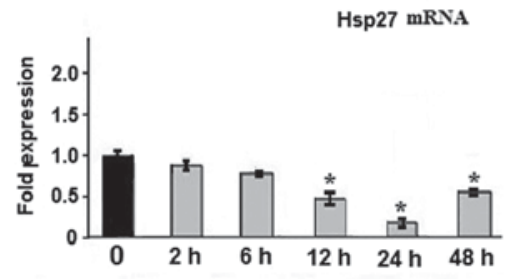

b

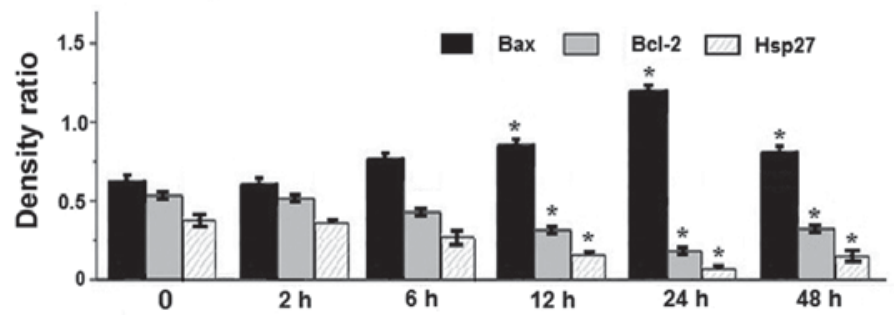

b

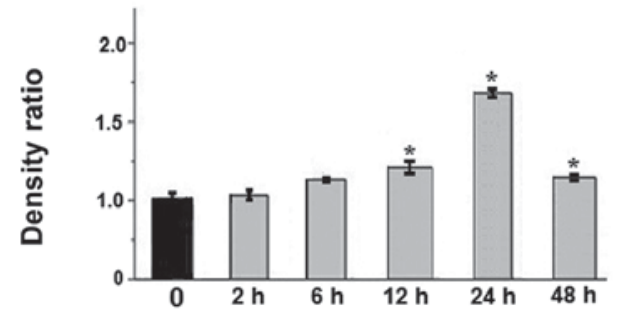

Figure 3. Iopamidol-induced HK2 cell damage and the expression of Bax/Bcl-2, cleaved/total caspase-3 and Hsp27 for different durations (2, 4, 6, 12, 24 and 48 h). (A) HK2 cell damage was assessed as viability (cell titer proliferation assay). (B) RT-qPCR results for the mRNA expression levels of Bax and Bcl-2 at different times. (C) RT-qPCR results for the mRNA expression of Hsp27 at different times. (Da) Western blot results for the protein expression of Bax/Bcl-2 and Hsp27. (Db) Graph of the results from five separate experiments. (Ea) Western blot results for the protein expression of cleaved/total caspase-3. (Eb) Graph of the results from five separate experiments. Data are presented as the mean \pm standard error of the mean $\left({ }^{*} \mathrm{P}<0.05\right.$, compared with $\left.\mathrm{CR}\right)$. RT-qPCR, reverse transcription-quantitative polymerase chain reaction; Bcl-2, B cell lymphoma-2, BAX, Bcl-2-associated X protein; CR, control rats; CA, atorvastatin (20 mg/kg/day); CM, iopamidol; CMA, iopamidol+atorvastatin (20 mg/kg/day).

compared with the CM group $(\mathrm{P}<0.01$; Fig. $1 \mathrm{C})$, whereas the percentage of apoptotic cells was significantly higher in the CM group, compared with the CMA group $(\mathrm{P}<0.05)$.

As shown in Fig. 2, the RT-qPCR and western blot analyses revealed the expression levels of Bax, Bcl-2 and cleaved caspase-3. The mRNA levels of Bax were increased in the CM group and decreased in CMA group (Fig. 2A), however, Bcl-2 was negatively correlated with Bax (Fig. 2B). Bax (Fig. 2Ca and b) and cleaved caspase-3 (Fig. 2Da and b) were significantly increased in the $\mathrm{CM}$ group $(\mathrm{P}<0.01)$, and decreased in the CMA group $(\mathrm{P}<0.05)$. However, the expression of $\mathrm{Bcl}-2$ was significantly decreased in the $\mathrm{CM}$ group and increased in the CMA group $(\mathrm{P}<0.05)$.

The exposure of HK2 cells to iopamidol induced a decrease in cell viability (Fig. 3A). This reaction was evident at $12 \mathrm{~h}$ and reached a peak $24 \mathrm{~h}$ following iopamidol treatment. It was also found that iopamidol increased the mRNA expression levels of Bax in a time-dependent manner (Fig. 3B), which was similar with the levels of cell viability. Notably, the mRNA level of Bcl-2 (Fig.3B) and Hsp27 (Fig.3C), and the protein expression levels of Bcl-2 and Hsp27 (Fig. 3Da and b) decreased at $12 \mathrm{~h}$ and reached a peak at $24 \mathrm{~h}$, which was negatively correlated with the levels of cell apoptosis. The protein expression of Bax increased in a time-dependent manner. Furthermore, caspase-3 activity was upregulated at $12 \mathrm{~h}$ and reached a peak at $24 \mathrm{~h}$ (Fig 3E).
Exposure of the HK2 cells to iopamidol also induced an increase in the activities of Bax (Fig. 4Aa and b) and caspase-3 (Fig. 4Ba and b), and a decrease in the activity of Bcl-2 (Fig. 4A). Following treatment with atorvastatin, the activities of Bax and caspase- 3 decreased, whereas that of Bcl-2 increased (Fig. 4A and B). TUNEL staining (Fig. 4Ca and b) was also more intense in the presence of iopamidol, and was weakened following atorvastatin treatment $(\mathrm{P}<0.05)$.

Protective effects of atorvastatin on CM-induced renal cell apoptosis through the upregulation of Hsp27. In the HK2 cells exposed to iopamidol, the mRNA and protein expression levels of Hsp27 were reduced at $12 \mathrm{~h}$, peaked at $24 \mathrm{~h}$ (Fig. 3C and D), and were negatively correlated with the levels of cell apoptosis.

Furthermore, although exposure of the HK2 cells to iopamidol induced a decrease in the level of Hsp27, treatment with atorvastatin resulted in an increase in the level of Hsp27 (Fig. 4A), which was negatively correlated with the levels of cells apoptosis in the TUNEL staining $(\mathrm{P}<0.05$; Fig. 4C).

As shown in Fig. 5Aa and b, immunohistochemical staining for Hsp27 was decreased in the CM group, but upregulated in the presence of atorvastatin. In addition, it was shown that $\mathrm{CM}$ induced a decrease in the mRNA levels of Hsp27 $(\mathrm{P}<0.05)$, and these increased significantly following atorvastatin treatment $(\mathrm{P}<0.05$; Fig. 5B). Similar changes were found in 
A

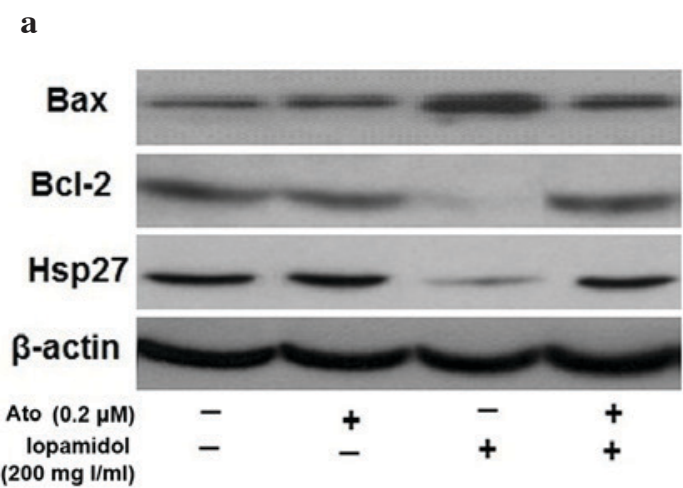

B

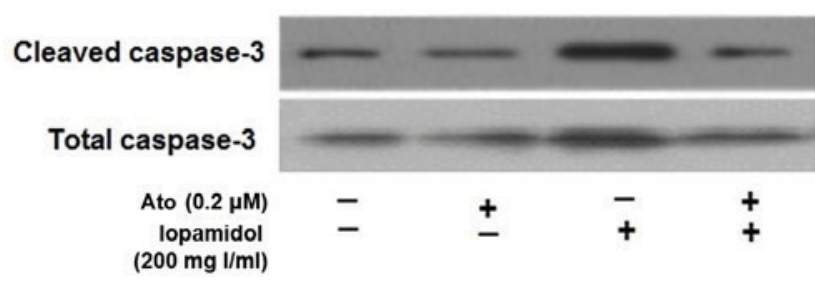

$\mathbf{C}$

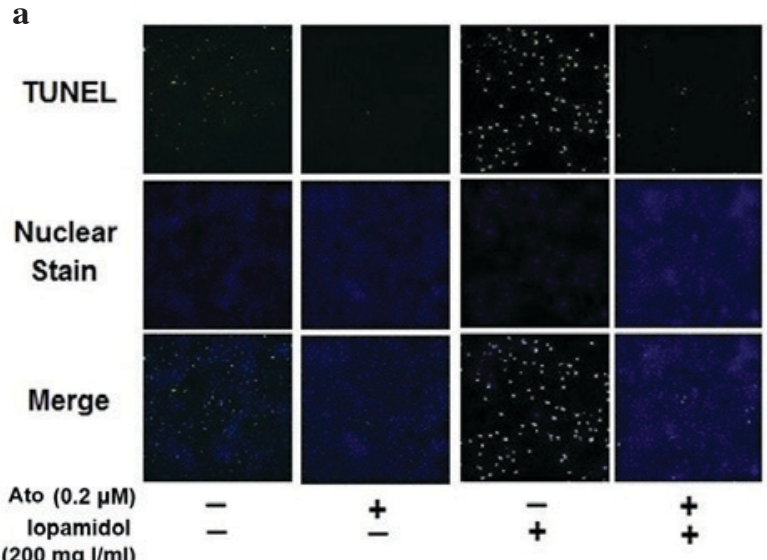

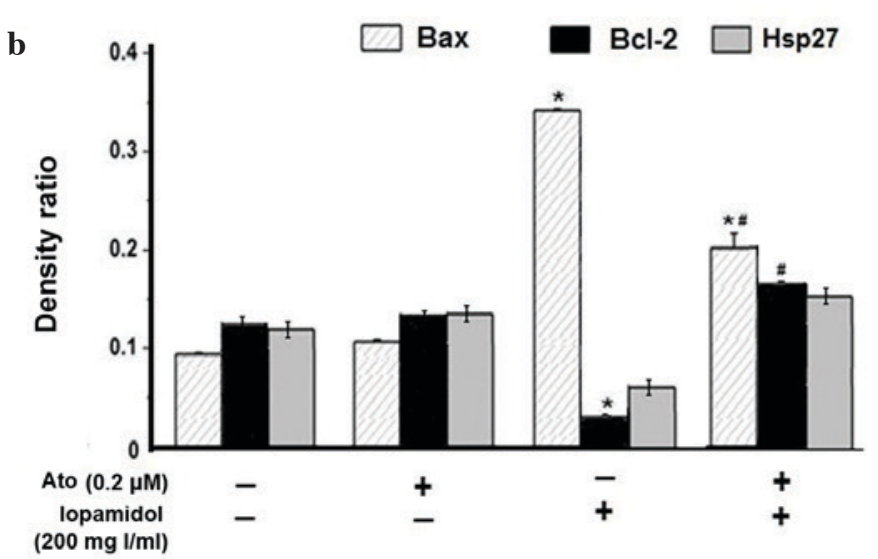

b

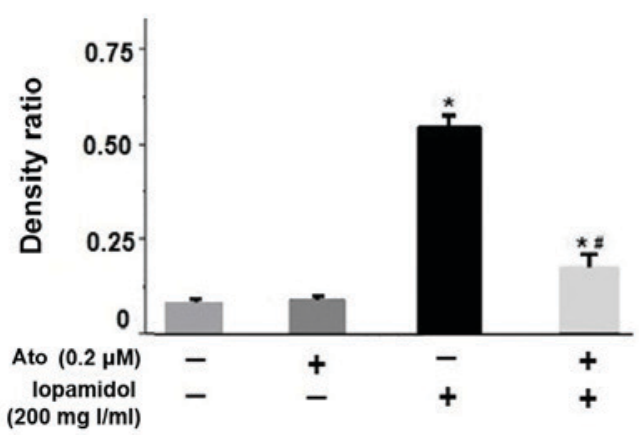

b

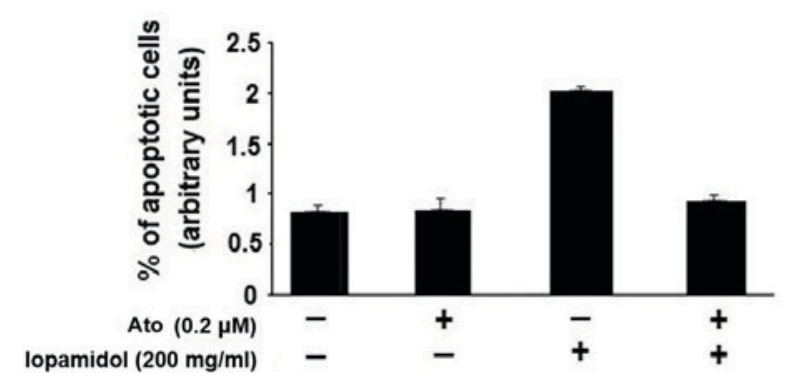

Figure 4. Effects of atorvastatin on the iopamidol-induced protein expression of Bax/Bcl-2 and cleaved/total caspase-3 in HK2 cells. (Aa) Western blot results for Bax/Bcl-2 and Hsp27 proteins. (Ab) Graph of the results from five separate experiments. (Ba) Western blot results for cleaved/total caspase-3 proteins. (Bb) Graph of the results from five separate experiments. (Ca) Representative images of TUNEL-positive renal tubular cell numbers (magnification, x200). ( $\mathrm{Cb})$ Graph of TUNEL-positive renal tubular cell numbers. Data are presented as the mean \pm standard error of the mean ("P<0.05, compared with the control; ${ }^{\text {"}} \mathrm{P}<0.05$, compared with iopamidol only). Bcl-2, B cell lymphoma-2, BAX, Bcl-2-associated X protein; Hsp27, heat shock protein 27.

Hsp27 protein via western blot analysis (Fig. 5Ca and b). The upregulation of Hsp27 was accompanied by improvements in levels of cell apoptosis, which was associated with a reduction in inflammatory markers and improvements in renal function (Tables II and III).

Following transfection with Hsp27 siRNA or the Hsp27 plasmid, it was found that the expression levels of Bax/caspase-3 (Fig. 5D and E) were increased and reduced by the inhibition and overexpression of Hsp27, respectively, with negative correlation with Bcl-2 (Fig. 5D), as detected using western blot analysis. Iopamidol also induced cell apoptosis (Fig. 5Fa and b). This suggested that the iopamidol-induced downregulation of Hsp27 may be one of the causes for HK2 cell apoptosis.

\section{Discussion}

The foundation of the prophylaxis of CIAKI is hydration; however, strategies to prevent CM-induced renal cell apoptosis appear to have indispensable clinical involvement. Previous clinical trials have been performed with the use of various antioxidant compounds with the aim of lowering the occurrence of CIAKI $(2,4)$. The present study showed the protective effect of atorvastatin on CM-induced renal cell damage. CIN is a type of renal injury caused, not only due to the toxicity of contrast media, but also due to renal ischemia, inflammatory reactions and cell apoptosis. According to the results of the present study, atorvastatin ameliorated renal injury, cell 
A a<smiles>[R]C1CCC1</smiles>
CA CM CMA b

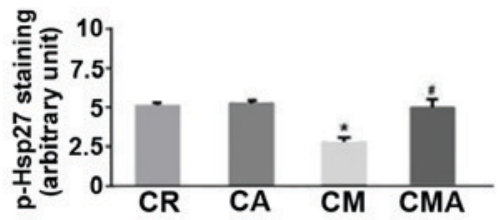

D

$\mathbf{a}$

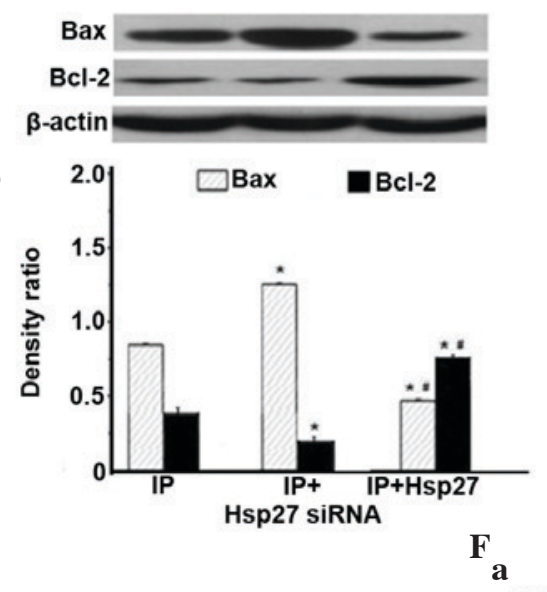

B

$\mathbf{E}$

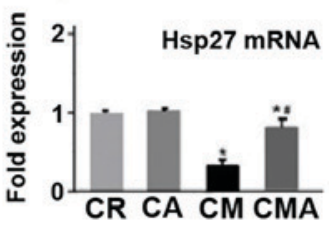

a
C a

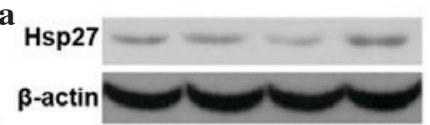

b

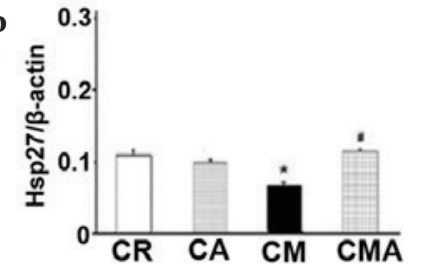

Cleaved caspase- 3
Total caspase- 3

b
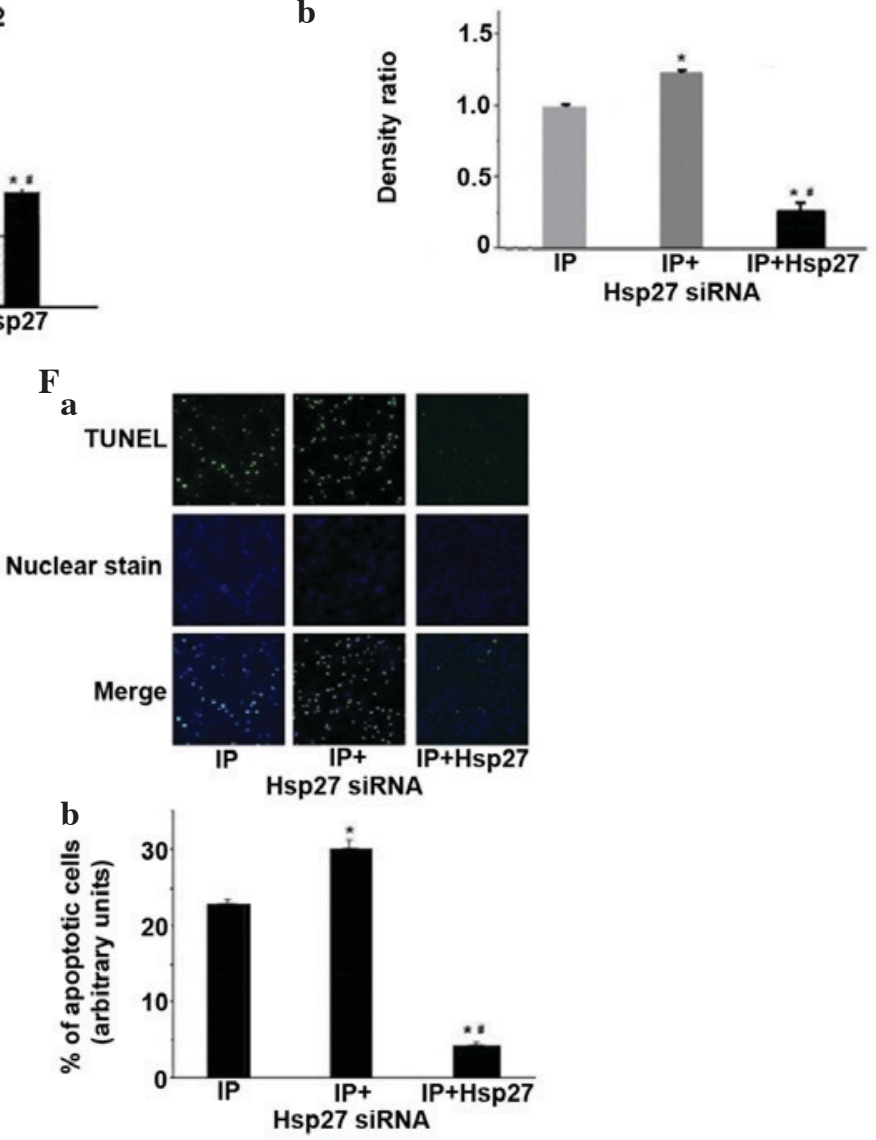

Figure 5. Effect of Hsp27 on CM-induced cell apoptosis in the presence or absence of atorvastatin. (Aa) Representative images of immunohistochemical staining for Hsp27 (magnification, x200). (Ab) Graph of the results from 10 separate experiments. (B) Reverse transcription-quantitative polymerase chain reaction analysis for the mRNA expression of Hsp27. (Ca) Western blot results for the Hsp27 protein, with a (Cb) graphical presentation of the results from 10 separate experiments. (Da and Ea) Western blot results for the expression of Bax/Bcl-2 and cleaved/total caspase-3 following transfection with Hsp27 siRNA and the Hsp27 plasmid. ( $\mathrm{Db}$ and $\mathrm{Eb}$ ) graphical presentation of the results from five separate experiments. (Fa) Representative images of TUNEL-positive renal tubular cell numbers (magnification, $\mathrm{x} 200$ ). (Fb) Graphical representation of TUNEL-positive renal tubular cell numbers. Data are presented as the mean \pm standard error of the mean. "P $<0.05$, compared with $\mathrm{CR}$ and IP; " $\mathrm{P}<0.05$, compared with $\mathrm{CM}$ and IP+Hsp27. CR, control rats; CA, atorvastatin (20 mg/kg/day); $\mathrm{CM}$, iopamidol; CMA, iopamidol+atorvastatin (20 mg/kg/day). IP, iopamidol (200 mgI/ml); IP+Hsp27 siRNA, iopamidol (200 mgI/ml)+Hsp27 siRNA (400 pmol); IP+Hsp27, iopamidol $(200 \mathrm{mgI} / \mathrm{ml})+\mathrm{pcDNA} 3.1-\mathrm{Hsp} 27(2 \mu \mathrm{g})$.

apoptosis and the deterioration of renal function. Furthermore, atorvastatin reduced the inflammatory reaction, suppressed caspase- 3 activity, and increased the expression of Bcl-2 in vivo and in vitro. Notably, the results of the present study suggested that iopamidol-induced HK2 cell apoptosis may be caused by the downregulation of Hsp27.

The inflammatory reaction is important in the pathogenesis of CIN. TNF- $\alpha$, IL- $1 \beta$ and IL- 6 are pro-inflammatory cytokines, which recruit numerous mediators associated with endothelial and tissue injury. In a mouse model of nephrotoxicity and in clinical trials, TNF- $\alpha$, IL- $1 \beta$ and IL- 6 have been shown to be central in activation of the inflammatory cytokine response $(11,16)$. The results of the present study further confirmed these previous findings, and demonstrated that the levels of inflammatory markers, TNF- $\alpha$, IL- $1 \beta$ and IL-6, were increased in the rats with CIN. Our previous 
studies and clinical investigations have shown that $\mathrm{SCr}$, urine microprotein and uKIM-1 are important indices for CIN prediction $(4,6,19)$. The present study further confirmed that $\mathrm{SCr}$, urine microprotein and uKIM-1 increased following iopamidol treatment.

Apoptosis is an important mechanism of acute kidney injury, including CIN. Suppressing apoptotic pathways may attenuate pathological alterations in CIN (7). It has been reported that mitochondrial and intrinsic apoptotic pathways are involved in the tubular injuries of CIN (8). Bax is pro-apoptotic protein, whereas $\mathrm{Bcl}-2$ is an anti-apoptotic protein, which can inhibit caspase-3. Increased levels of Bcl-2 and decreased levels caspase- 3 activity may contribute to reduced apoptosis $(7,8,20)$. In the present study, renal tubular cell apoptosis was observed in the CIN kidney, consistent with previous results. In addition, increased Bax/caspase-3 activity and decreased expression levels of Bcl-2 were detected in the CIN rats and HK-2 cells treated with iopamidol. Thus, iopamidol-induced cell apoptosis may be associated with activation of Bax/caspase-3 and inhibition of Bcl-2.

Statins reduce the levels of intracellular pro-inflammatory factors in several cellular systems by acting on the inhibition of producing enzymes $(12,21,22)$. In the rat model used in the present study, pretreatment with atorvastatin reduced the iopamidol-induced release of TNF- $\alpha$, IL-1 $\beta$ and IL- 6 , and improved renal function, including the levels of $\mathrm{SCr}$, urine microprotein and $\mathrm{uKIM}-1$. In addition, the increased expression of Bax/caspase-3 and decreased expression of Bcl-2 were partially reversed by atorvastatin in the CIN rats and HK 2 cells treated with iopamidol. Accordingly, the protective effect of atorvastatin in CIN may be associated with the $\mathrm{Bcl}-2 /$ caspase-3 anti-apoptotic pathway.

Hsp27 is a small HSP, which is important in stress tolerance in several cellular processes. The local tissue expression of Hsp27 reduces the levels of proinflammatory cytokines, including TNF- $\alpha$, and protects cells the against oxidative stress caused by renal tubular cell apoptosis in ischemia-induced acute kidney injury $(6,19,23)$. However, whether Hsp27 has a protective effect in CIN, and the underlying molecular mechanism, remain to be elucidated. In the present study, western blot analysis demonstrated that Hsp27 was upregulated in the CMA rats and downregulated in the CM rats, which was negatively correlated with the expression of Bax/caspase- 3 and the levels of apoptosis in the HK2 cells. Hsp27 inhibits the release of caspase- 3 and the gene expression of Bax, which leads to reduced apoptosis and organ injury. Following treatment with atorvastatin, Hsp27 was upregulated, together with improvements in HK2 cell apoptosis. Furthermore, through transfection with Hsp27 siRNA and the Hsp27 plasmid, it was found that iopamidol induced cell apoptosis, and that the activity of Bax/caspase- 3 was enhanced and reduced by inhibition and overexpression of Hsp27, respectively. These findings suggested that iopamidol aggravated renal tubular injury via the downregulation of activated Hsp27, which weakened its anti-apoptotic effects, resulting in an increase in renal tubular apoptosis.

In conclusion, the data obtained in the present study suggested that atorvastatin exhibited renal protection in the CIN model via the anti-inflammatory reaction and upregulation of Hsp27.

\section{Acknowledgements}

The present study was supported by the Special Fund for Basic Research on Scientific Instruments, National Natural Science Foundation, China (grant no. 81470571) and the 2014 Guangzhou Project of Science and Technology (grant no. 2014y2-00140).

\section{References}

1. Nash K, Hafeez A and Hou S: Hospital-acquired renal insufficiency. Am J Kidney Dis 39: 930-936, 2002.

2. Schilp J, de Blok C, Langelaan M, Spreeuwenberg P and Wagner C: Guideline adherence for identification and hydration of high-risk hospital patients for contrast-induced nephropathy. Bmc Nephrology 15: 2, 2014.

3. Jorgensen AL: Contrast-induced nephropathy: Pathophysiology and preventive strategies. Crit Care Nurse 33: 37-46, 2013.

4. Liu Y, Tan N, Chen J, Zhou Y, Chen L, Chen S, Chen Z and $\mathrm{Li}$ L: The relationship between hyperuricemia and the risk of contrast-induced acute kidney injury after percutaneous coronary intervention in patients with relatively normal serum creatinine. Clinics (Sao Paulo) 68: 19-25, 2013.

5. Rundback JH, Nahl D and Yoo V: Contrast-induced nephropathy. J Vasc Surg 54: 575-579, 2011.

6. Liu YH, Liu Y, Tan N, Chen JY, Zhou YL, Luo JF, Yu DQ, Li LW, Li HL, Ye P and Ran P: Contrast-induced nephropathy following chronic total occlusion percutaneous coronary intervention in patients with chronic kidney disease. Eur Radiol 25: 2274-2281, 2015.

7. Buyuklu M, Kandemir FM, Ozkaraca M, Set T, Bakirci EM and Topal E: Protective effect of curcumin against contrast induced nephropathy in rat kidney: What is happening to oxidative stress, inflammation, autophagy and apoptosis? Eur Rev Med Pharmacol Sci 18: 461-470,2014.

8. Quintavalle C, Brenca M, De Micco F, Fiore D, Romano S, Romano MF, Apone F, Bianco A, Zabatta MA, Troncone G, et al: In vivo and in vitro assessment of pathways involved in contrast media-induced renal cells apoptosis. Cell Death Dis 2: e155, 2011.

9. Al-Otaibi KE, Al Elaiwi AM, Tariq M and Al-Asmari AK: Simvastatin attenuates contrast-induced nephropathy through modulation of oxidative stress, proinflammatory myeloperoxidase and nitric oxide. Oxid Med Cell Longev 2012: 831748, 2012.

10. Patti G, Ricottini E, Nusca A, Colonna G, Pasceri V, D'Ambrosio A, Montinaro A and Di Sciascio G: Short-term, high-dose atorvastatin pretreatment to prevent contrast-induced nephropathy in patients with acute coronary syndromes undergoing percutaneous coronary intervention (from the armyda-cin (atorvastatin for reduction of myocardial damage durin during angioplasty-contrast-induced nephropathy) trial. Am J Cardiol 108: 1-7, 2011.

11. Quintavalle C, Fiore D, DeMicco F, Visconti G, Focaccio A, Golia B, Ricciardelli B, Donnarumma E, Bianco A, Zabatta MA et al: Impact of a high loading dose of atorvastatin on contrast-induced acute kidney injury. Circulation 126: 3008-3016, 2012.

12. Patti G, Nusca A, Chello M, Pasceri V, D'Ambrosio A, Vetrovec GW and Di Sciascio G: Usefulness of statin pretreatment to prevent contrast-induced nephropathy and to improve long-term outcome in patients undergoing percutaneous coronary intervention. AM J Cardiol 101: 279-285, 2008.

13. Bakthisaran R, Tangirala R and Rao CM: Small heat shock proteins: Role in cellular functions and pathology. Biochim Biophys Acta 1854: 291-319, 2015.

14. Acunzo J, Andrieu C, Baylot V, So A and Rocchi P: Hsp27 as a therapeutic target in cancers. Curr Drug Targets 15: 423-431, 2014.

15. Clark JD, Gebhart GF, Gonder JC, Keeling ME and Kohn DF: Special Report: The 1996 Guide for the Care and Use of Laboratory Animals. ILAR J 38: 41-48, 1997.

16. Inci MF, Salk I, Solak O, Vurdem UE and Inci R: Use of $\mathrm{N}$-acetylcysteine for the prevention of contrast-induced nephropathy in rats. Actas Urol Esp 36: 210-215 (In Spanish), 2012. 
17. Livak KJ and Schmittgen TD: Analysis of relative gene expression data using real-time quantitative PCR and the 2(-Delta Delta C(T)) Method. Methods 25: 402-408, 2001.

18. He X, Gao X, Peng L, Wang S, Zhu Y, Ma H, Lin J and Duan DD: Atrial fibrillation induces myocardial fibrosis through angiotensin II type 1 receptor-specific Arkadia-mediated downregulation of Smad7. Cir Res 108: 164-175, 2011.

19. Lauta VM: Interleukin- 6 and the network of several cytokines in multiple myeloma: An overview of clinical and experimental data. Cytokine 16: 79-86, 2001.

20. Ahmad A, Mondello S, Di Paola R, Mazzon E, Esposito E, Catania MA, Italiano D, Mondello P, Aloisi C and Cuzzocrea S: Protective effect of apocynin, a NADPH-oxidase inhibitor, against contrast-induced nephropathy in the diabetic rats: A comparison with n-acetylcysteine. Eur J Pharmacol 674: 397-406, 2012.
21. Su J, Zou W, Cai W, Chen X, Wang F, Li S, Ma W and Cao Y: Atorvastatin ameliorates contrast medium-induced renal tubular cell apoptosis in diabetic rats via suppression of Rho-kinase pathway. Eur J Pharmacol 723: 15-22, 2014.

22. Riad A, Du J, Stiehl S, Westermann D, Mohr Z, Sobirey M, Doehner W, Adams V, Pauschinger M, Schultheiss HP and Tschöpe C: Low-dose treatment with atorvastatin leads to anti-oxidative and anti-inflammatory effects in diabetes mellitus. Eur J Pharmacol 569: 204-211, 2007.

23. Chang CF, Lu TM, Yang WC, Lin SJ, Lin CC and Chung MY: Gene polymorphisms of interleukin-10 and tumor necrosis factor- $\alpha$ are associated with contrast-induced nephropathy. Am J Nephrol 37: 110-117, 2013. 